\title{
Towards Modeling Partial Discharge Phenomena and Propagation in Power Networks Using the Transmission-Line Matrix Method
}

\author{
Antonella Ragusa *, Hugh Sasse and Alistair Duffy \\ Faculty of Technology, De Montfort University, Leicester LE1 9BH, UK; hgs@dmu.ac.uk (H.S.); \\ apd@dmu.ac.uk (A.D.) \\ * Correspondence: antonella.ragusa@dmu.ac.uk
}

check for

updates

Citation: Ragusa, A.; Sasse, H.;

Duffy, A. Towards Modeling Partial

Discharge Phenomena and

Propagation in Power Networks

Using the Transmission-Line Matrix

Method. Energies 2021, 14, 689.

https://doi.org/10.3390/en14030689

Academic Editor: Zheng Xu

Received: 5 January 2021

Accepted: 26 January 2021

Published: 29 January 2021

Publisher's Note: MDPI stays neutral with regard to jurisdictional claims in published maps and institutional affiliations.

Copyright: (C) 2021 by the authors. Licensee MDPI, Basel, Switzerland. This article is an open access article distributed under the terms and conditions of the Creative Commons Attribution (CC BY) license (https:// creativecommons.org/licenses/by/ $4.0 /)$.

\begin{abstract}
Partial discharge (PD), where high field strengths on power cables cause charge build up and discharge within a dielectric at sites of imperfections or inhomogeneities, can lead to noise issues and potential failure of the dielectric. This paper presents the first stage of a research activity that aims to develop a transmission-line matrix (TLM)-based simulation "workbench" useful to investigate PD events in a transmission line. The proposed approach allows the predicting of the electromagnetic disturbances generated by the PD event, and the analysis of external field coupling, such as from intentional electromagnetic interference or lightning, which can add to the field stresses. The paper is focused on defining the right modeling method to simulate PD phenomena in a transmission line context. The best approach to integrate the PD model with the model of the transmission line, useful to describe the propagation of the conducted and radiated emissions produced by PD, is analyzed. A first workbench is proposed, and the first simulation results are described. The paper concludes with the topics of further research.
\end{abstract}

Keywords: partial discharge; power cables; electromagnetic interference; transmission line matrix modeling

\section{Introduction}

The nature of partial discharge (PD) is such that should a local field strength in a power cable or switchgear be sufficient to exceed the local dielectric strength, then a localized "spark" will occur. This charge movement within the body of the dielectric may occur in one small region due to the presence of inhomogeneities such as oil or gas bubbles, or localized changes in the structure of the material (perhaps the "clumping" of additives or micro-cracks) causing charge build-up around this imperfection due to electric field effects. If the local field strength exceeds the dielectric strength of the imperfection, then a localized breakdown will occur. Other causes include cavities, electrical trees, and protrusions from semi-conducting layers [1].

The effect of this breakdown can be conducted or radiated electromagnetic noise, which may cause issues with, for example, smart grid timing signals or non-linear loads. The further deterioration of the insulator in the region of that breakdown could lead to further PDs, as well as an increased likelihood of total failure of the dielectric [2], causing the interruption of power supply with a reduction in network reliability [3]. Statistics indicate that more than $85 \%$ of equipment failures are related to insulation failure; therefore, managing the insulation of cables or equipment is crucial to avoid unnecessary emergency repairs (and cope with the effects of un-planned down-time). In order to define the insulation condition assessment of power networks, suitable PD measurement techniques have been developed that provide information about the defect types, their quantification, and the risk of failure that they can produce [3-6]. The use of a PD on-line location method is a desired feature in modern grids' protection schemes to prevent faults and 
supply interruption, enhancing the grids resilience [7-11]. Then, from the perspective of the electromagnetic compatibility (EMC) community, monitoring and analyzing PD is of direct relevance and, in particular, the nature of interference signals caused by PD is of interest. Zhang et al. [12-14] consider more discharge phenomena from power systems but, importantly, the impact that these can have on "internet of things" (IoT) and "smart grid" (SG) systems. It is likely that the importance of this will only increase with time as the importance of IoT and SG increases. The study of the PD electromagnetic wave emissions is of interest, also because they are used in the most adopted PD location methods, which are reflectometry or traveling wave-based techniques [7-11].

This paper proposes a simulation "workbench", useful to investigate both the PD site in a power transmission line and the generated electromagnetic emission.

Modeling of PD events is an active research area. PD modeling is, frequently, performed mathematically, and this can be restrictive, not readily accounting for effects due to dispersive behavior of the power cable or multiple PD locations. PD models proposed in the literature can be classified into three main categories [15]: (i) the three-capacitance model [16]; (ii) the analytical based model proposed by Niemeyer [17]; and (iii) the finite element analysis (FEA) [18] model. Each modeling method is characterized by its own strengths and weaknesses [15]. A well accepted model of PD is that the site of the discharge is treated as a capacitor, $\mathrm{Cc}$, with a shorting switch (with further series capacitors, $\mathrm{Cb} 1$ and $\mathrm{Cb} 2$, representing the unaffected dielectric), as illustrated in Figure 1, where the middle capacitor with the shunt switch models the discharge site in a transmission (Tx in the figure) line.

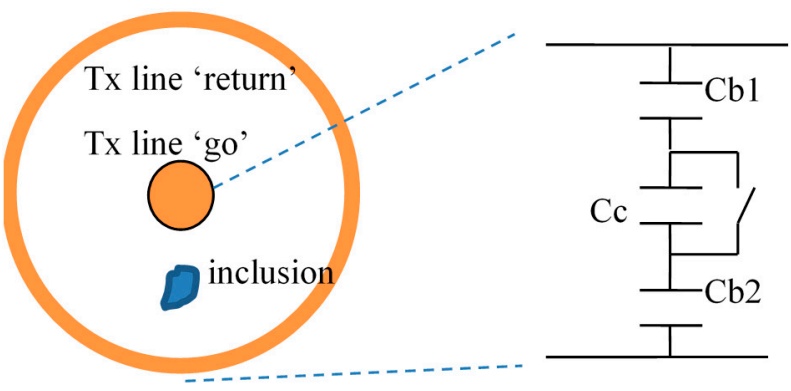

Figure 1. Series capacitor model of a partial discharge site, where the switch is closed at the point of discharge.

This is, essentially, a 2D view of the problem space. However, as explained before, there are benefits in considering the transmission line as a whole, because this allows questions to be asked and investigated. Such as: given that the PD will generate high frequency components and those may both radiate and propagate along a potentially frequency-dispersive transmission line, what effects are measurable? If this were to be undertaken analytically, there would be little scope for customization and asking questions, such as: how does the nature of the PD affect the measured effects?; or even: can the final measurements be time-reversed to understand the location and nature of the original problem? In the literature, few works exist that describe PD signal propagation on power networks [19-21], but a full model that reproduces both the disturbance source (PD site), and the propagation of the generated disturbance has not been investigated.

Given that the very nature of the problem is vastly multi-scale (with microscopic flaws giving rise to effects measurable meters or kilometers away), a full wave solution is not generally possible. Given also that this is a time domain phenomenon, a time domain simulation method is the most suitable. For the purpose of this study, the transmission-line matrix (TLM) method [22] has been adopted, because it allows both a circuit-based implementation as well as being naturally suited to modeling full transmission lines. This paper first investigates a circuit-based implementation (Figure 1) and explores strengths and weaknesses of that approach before investigating the simulation of a longer line. To this aim, a TLM model of the PD site and of the transmission line was developed and the 
propagation of the PD pulses was described, and the effects of radiation due to the pulse propagation were explored.

The paper is structured as follows. Section 2 describes the best approach to model PD events in a transmission line context, describing the TLM model of a PD site. Section 3 describes the integration of the TLM PD model with a 1D TLM model of the transmission line showing the effectiveness in reproducing the propagation of PD-generated pulses along the network; Section 4 describes a method to evaluate radiated emission from PD pulses. In Section 5, an illustration of the use of the proposed workbench to describe the conducted and radiated emissions produced by a PD event occurring in a medium voltage transmission line is presented. Finally, Section 6 presents the conclusions, the strengths of the proposed workbench, the necessary steps to improve it to obtain a better representation of the phenomena under study, and the work under development to reach this aim.

\section{Transmission Line Matrix Representation of the Partial Discharge Site}

A very common representation of partial discharge in a power cable is presented in Figure 1; Figure 2 shows how the three-capacitor ladder can be included in a general Thevenin-based equivalent circuit, suitable for analysis.

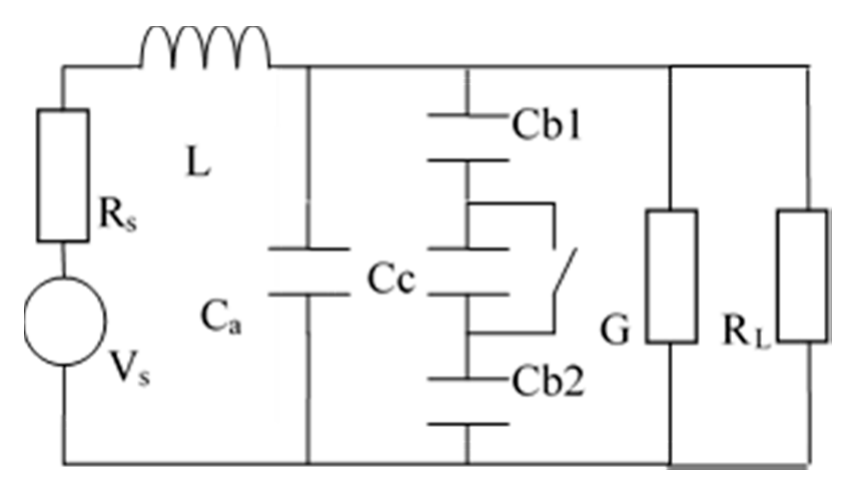

Figure 2. A general representation of a partial discharge site within a transmission line.

Here, a typical RLGC transmission line element is shown, where $\mathrm{R}$ and $\mathrm{L}$ are the series resistance and inductance of the line and $G$ and $C$ are the shunt conductance and capacitance. The capacitor term is replaced by a capacitance representing the bulk material $\left(\mathrm{C}_{\mathrm{a}}\right)$ with the capacitance of the partial discharge site, such as a void, represented by a capacitor $(\mathrm{Cc})$ in parallel with a switch. The closing of the switch represents the partial discharge event where the charge stored in the capacitor $\mathrm{Cc}$ neutralizes through a localized discharge. The "normal" dielectric, either side of the discharge site, is represented by two capacitors $(\mathrm{Cb} 1$ and $\mathrm{Cb} 2) . \mathrm{V}_{\mathrm{S}}$ and $\mathrm{R}_{\mathrm{S}}$ represent a Thevenin source, and the transmission line is terminated in a load $\mathrm{R}_{\mathrm{L}}$. There is a body of work that looks to calculate the value of Cc by analysis or simulation, e.g., $[1,17,23]$.

The PD site can be represented, adopting the simple circuit-based TLM method that uses transmission line equivalent behavior to represent circuital components such as capacitors and inductors. A capacitor is replaced by an open circuit transmission line stub and an inductor by a short circuit transmission line stub. As the simulation progresses, with voltages and currents propagating in small increments of time to simulate any time-domain or transient behavior of the circuit, the capacitive open (or inductive short) circuit reflects voltages on the stub back to the terminals. This is represented by an impedance in series with a voltage source.

Figure 3 gives a generic representation of a component. 


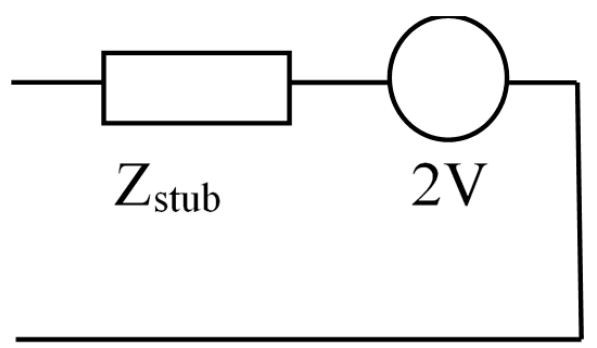

Figure 3. Generic representation of a circuit element using the transmission-line matrix (TLM).

For a stub representation of the inductance and capacitance, the value of the impedance terms, $Z_{L}$ and $Z_{C}$, is given by Equations (1) and (2), respectively, where $\Delta t$ is the time step duration and $C$ and $L$ are the values of the nodal capacitance and inductance:

$$
\begin{aligned}
& Z_{C}=\Delta t / 2 C \\
& Z_{L}=2 L / \Delta t
\end{aligned}
$$

The voltage across the capacitor, $\mathrm{V}_{\text {stub }}$, is given by Equation (3), where $\mathrm{I}$ is the current flowing through the stub, and V is the voltage source value (as shown in Figure 2). Assuming that the reflection coefficient, $\Gamma$, at the end of the stub is +1 ( -1 for an inductor), then the value of $\mathrm{V}$ for the next iteration is given by Equation (4):

$$
\begin{gathered}
\mathrm{V}_{\text {stub }}=\mathrm{I} \cdot \mathrm{Z}_{\mathrm{C}}+2 \mathrm{~V} \\
\mathrm{~V}_{\text {(next time step) }}=\mathrm{V}+\Gamma \cdot \mathrm{I} \cdot \mathrm{Z}_{\mathrm{C}}
\end{gathered}
$$

The new current and new voltages can then be calculated before proceeding to the next iteration. The assembled representation of Figure 2 can be seen in Figure 4.

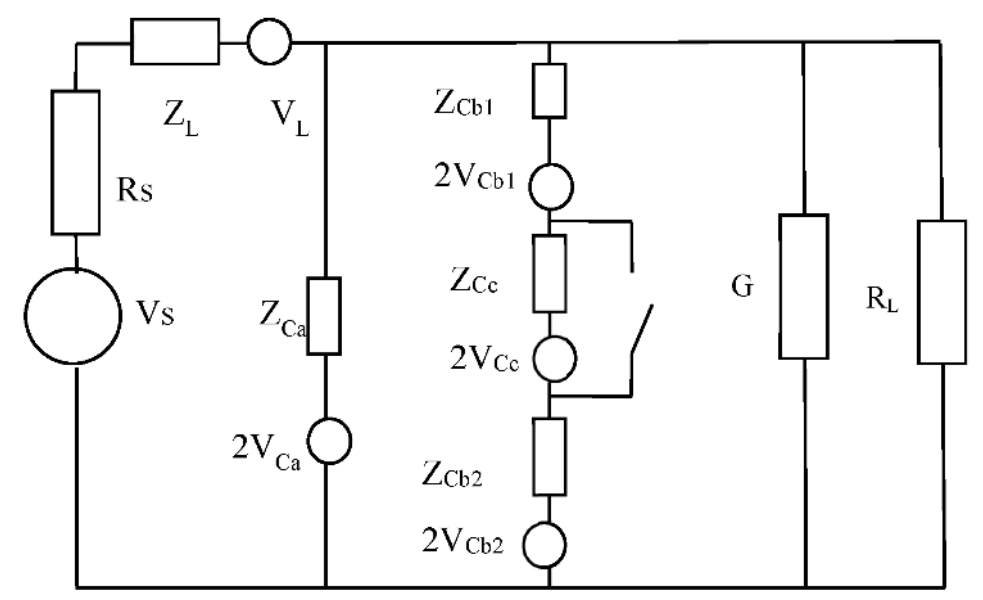

Figure 4. TLM representation of the partial discharge site.

However, a simple implementation such as this demonstrated instability where a mesh-based analysis led to inconsistencies with Kirchhoff's laws. This is something that may be resolvable with very small time-steps, as discussed in [24]. Such a restriction would not be practical.

A more stable approach is to treat the three capacitors as a single impedance, $Z_{p d}$ and voltage source, $V_{\mathrm{pd}}$. A basic circuit for this is given in Figure 5, where the variable impedance includes the switch function itself. 


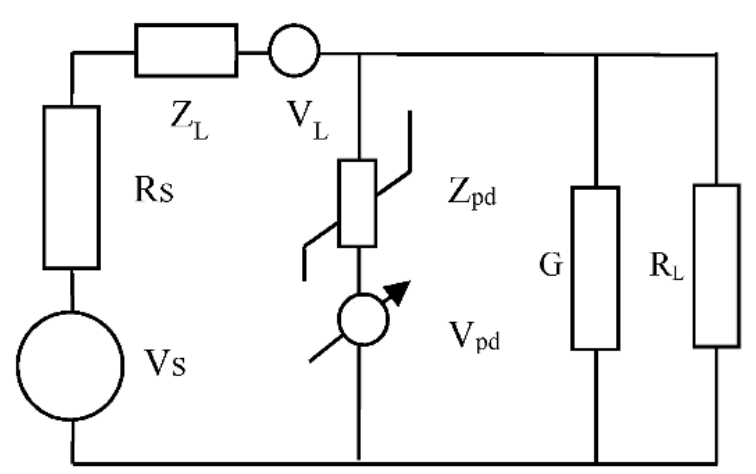

Figure 5. Equivalent representation of the lumped series capacitors.

When implemented with a $\Delta t$ of the order of one-tenth of a cycle and not many orders of magnitude lower, and a simple decision condition of triggering the event when the electric field across the central "PD" capacitor exceeded a certain level, the current flowing in the circuit can be seen in Figure 6. It is clear that such a circuit can help illustrate the behavior of a partial discharge event (even with such a crude decision strategy as was implemented here).

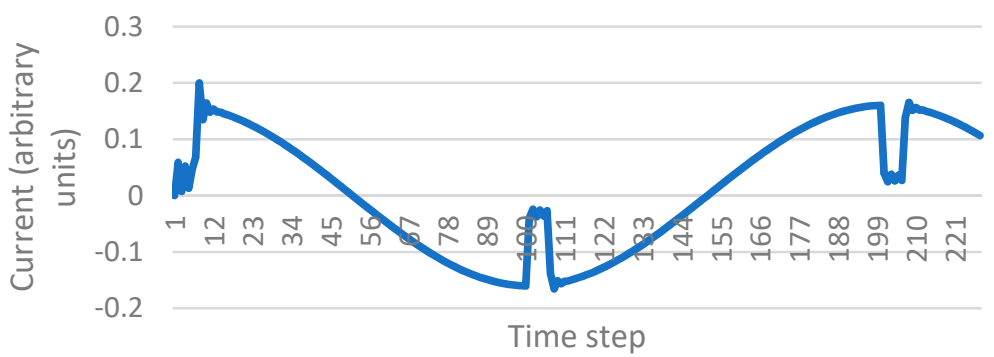

Figure 6. Circuit current for an example of the implementation of the simple partial discharge circuit.

There is still a need to add flexibility to the approach, and the simple circuit model is not investigated further. A transmission-line matrix representation of the entire transmission line in which the partial discharge occurs is required, where flexibility in the way that the partial discharge is included is available. The model shown above could be one such approach to embedding these phenomena. Equally simple pulse injection, deterministic equations, embedded simulations, or actual measurements in the form of a "look up" table should equally be possible. The next sections look at the development and subsequently the initial results of a 1D TLM representation.

\section{1D Transmission-Line Matrix Representation of a Line with Partial Discharge Site}

As previously noted, the 1D TLM method is well suited to modeling transmission lines such as power distribution lines. The relative ease in which distributed sources can be included in the model makes the approach a relatively straightforward choice for problems including coupling from external fields or internal sources.

In 1D TLM, space and time are both discretized, with each segment, or node, representing an electrically (very) small length of the space being modelled. Voltages are received from the left and right connected segments. The Millman's parallel generator theorem can be used to solve the resulting nodal circuit, evaluating the new voltages at the left and right side of the segment. These "scattered" pulses "connect" to the adjacent segments in the next time step. A complete transmission line can be modelled, where each segment (or node) can be also characterized by properties slightly different from its neighbors, giving rise to lengthwise variations. PD events can be included, in the manners previously described, at any node by adding voltage and/or current sources. The model could include evolution of the partial discharge source over time as a result of effects of 
the discharge on that site. High frequency signals or signatures can be obtained simply by using the Fourier transform. The frequency dependent behavior can be represented by using, within each of the segments, digital filters or Z-transforms. In this first study, the frequency dependent behavior was not considered.

A starting point for analysis of the system is a basic RLCG equivalent of each segment, as shown in Figure 7.

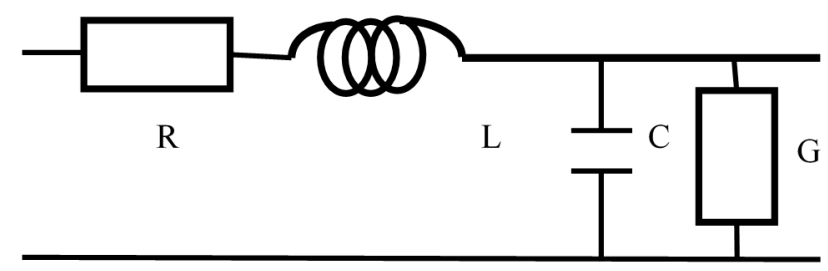

Figure 7. RLCG equivalent of lumped parameter equivalent circuit.

For the purpose of analysis, each transmission line segment can be thought of as connecting within a node, and it is here that the scatter process takes place. Such a node is shown in Figure 8.

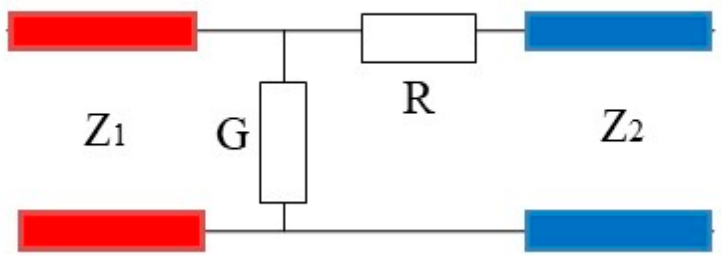

Figure 8. A nodal representation of two connected transmission lines.

Clearly, signals entering the node from the left side would do so via $Z_{1}$; they would then interact with $G, R$, and any impedance difference due to $Z_{2}$. A similar scatter process would occur for signals coming from the right side. Once the signals have scattered and passed through the node, they will "connect" to the adjacent nodes forming the initial conditions in the next time increment.

It is then possible to solve the circuit of Figure 8 using the Thevenin equivalent circuit of Figure 9. The subscript " $\mathrm{i}$ " refers to "incident", " 1 " and " $\mathrm{r}$ " as second subscripts mean left and right, respectively.

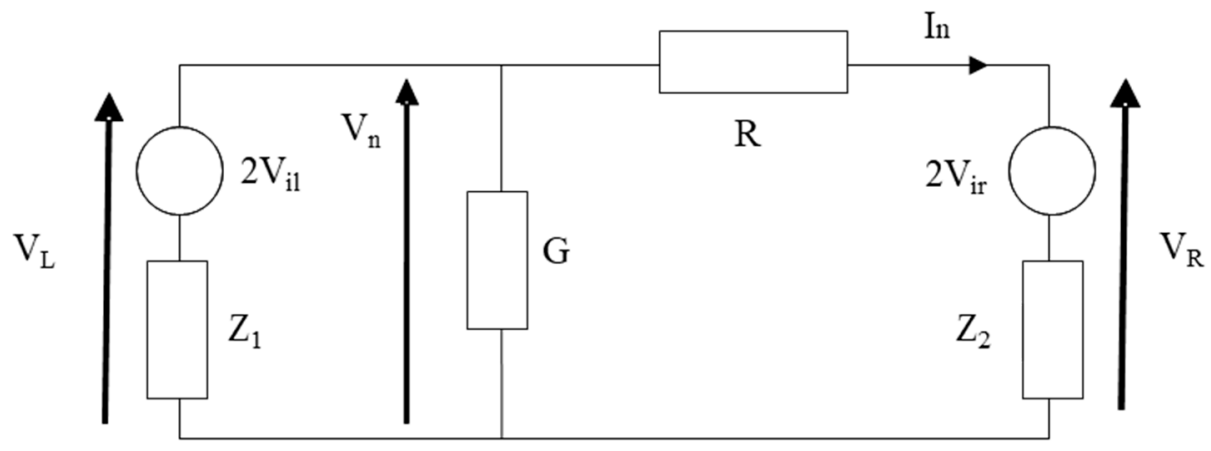

Figure 9. Thevenin equivalent circuit.

In Figure $9, V_{n}$ is the voltage node, $V_{L}$ and $V_{R}$ are the node voltage on the left and on the right of the node, respectively, $I_{n}$ is the current at the node, $R$ and $G$ are the resistance and conductance of the node, respectively, $Z_{1}$ and $Z_{2}$ are the impedance of the line on the left and on the right of the node, respectively, and $V_{11}$ and $V_{1 r}$ are the voltages of the Thevenin equivalent circuit of the line on the left and on the right of the node, respectively. 
Applying Millman's parallel generator theorem to Figure 9 enables the nodal voltage to be obtained from Equation (5); the potential difference on the left side can be obtained from Equation (6) with the right branch circulating current in Equation (7); and the righthand potential difference is given by Equation (8).

$$
\begin{gathered}
\mathrm{V}_{\mathrm{n}}=\frac{\frac{2 \mathrm{~V}_{\mathrm{il}}}{\mathrm{Z}_{1}}+\frac{2 \mathrm{~V}_{\text {ir }}}{\mathrm{Z}_{2}+\mathrm{R}}}{\frac{1}{\mathrm{Z}_{1}}+\frac{1}{\mathrm{R}+\mathrm{Z}_{2}}+\mathrm{G}} \\
\mathrm{V}_{\mathrm{L}}=\mathrm{V}_{\mathrm{n}} \\
\mathrm{I}_{\mathrm{n}}=\frac{\mathrm{V}_{\mathrm{n}}-2 \mathrm{~V}_{\mathrm{ir}}}{\mathrm{R}+\mathrm{Z}_{2}} \\
\mathrm{~V}_{\mathrm{R}}=2 \mathrm{~V}_{\mathrm{ir}}+\mathrm{I}_{\mathrm{n}} \mathrm{Z}_{2}
\end{gathered}
$$

Source and load nodes would have one branch of the node replaced by an equivalent source or a load. The relevant voltage and current values can be found by using Equations (5)-(7).

Having described the general node, a partial discharge event can be included in the node as an additional source term. A voltage source can be added in series with other sources. A current source, $\mathrm{I}_{\mathrm{pd}}$, can be included as indicated as in Figure 10, with the modified nodal voltage as given in Equation (9).

$$
V_{n}=\frac{\frac{2 V_{i l}}{Z_{1}}+\frac{2 V_{i r}}{Z_{2}+R}+I_{p d}}{\frac{1}{Z_{1}}+\frac{1}{R+Z_{2}}+G}
$$

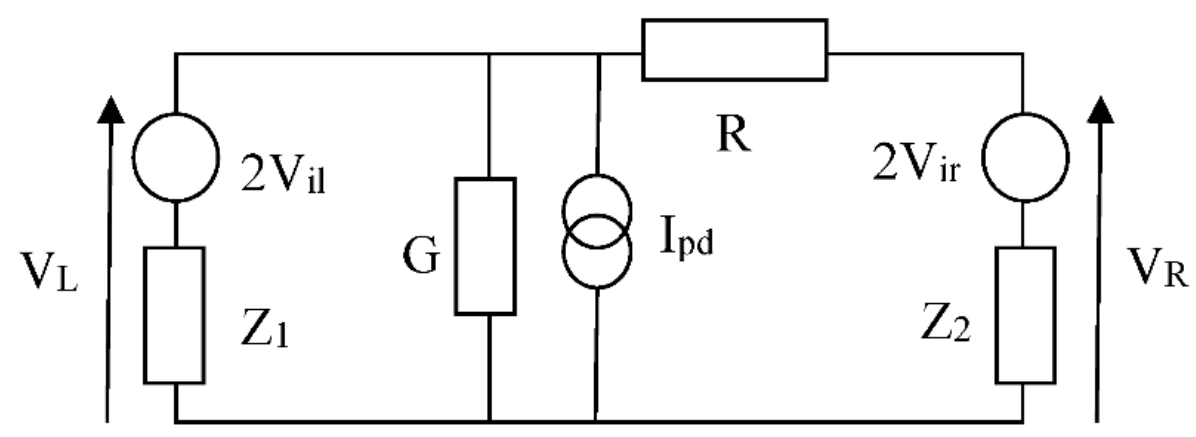

Figure 10. Current source added to a nodal equivalent circuit.

Once the signals have scattered through the node, they connect to the adjacent nodes according to Equations (10) and (11), where the subscript " $r$ " indicates a reflected signal.

$$
\begin{aligned}
& \mathrm{V}_{\mathrm{rl}}=\mathrm{V}_{\mathrm{L}}-\mathrm{V}_{\mathrm{il}} \\
& \mathrm{V}_{\mathrm{rr}}=\mathrm{V}_{\mathrm{R}}-\mathrm{V}_{\mathrm{ir}}
\end{aligned}
$$

This section has described how the nodes can be analyzed and used to simulate transmission lines with included source nodes. The performance can be seen in the following illustrations.

In order to demonstrate the general operation of the method, a pulse has been injected at the near end of a 50,000-node transmission line. The load and the source were not perfectly matched to the line, which allowed reflection. The line was lossy. In order to show the pulse propagation, attenuation and manage the local memory adequately, periodic samples were taken to show the passage of the pulses.

The axis system definition is shown in Figure 11. 


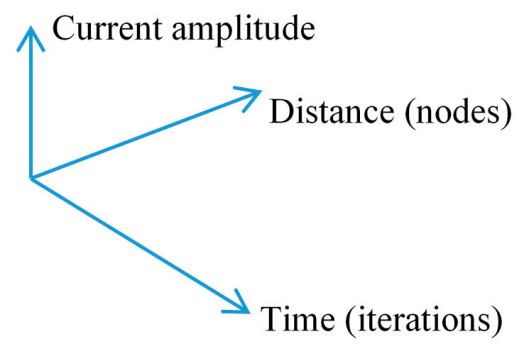

Figure 11. Axis definition for the following illustrations.

Figure 12 shows a simple pulse propagating along the transmission line. The "peakiness" is because of illustration samples being taken periodically. The illustration shows reflection at the load (far) end as well as attenuation as the pulse propagates along the transmission line over time.

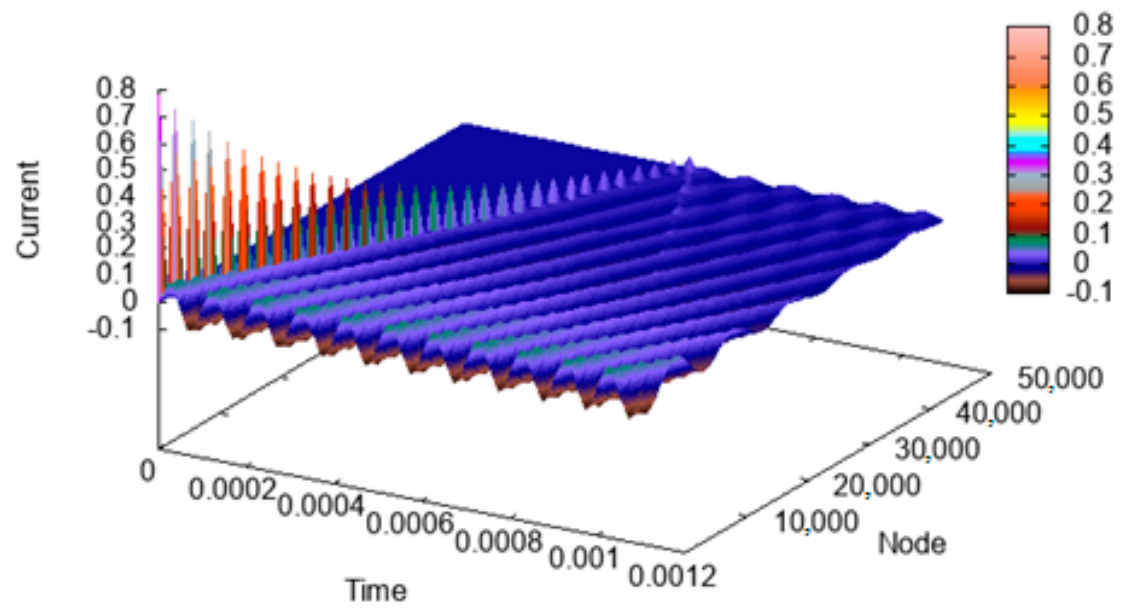

Figure 12. Demonstration of simple pulse propagating in both time and distance.

Figure 13 shows the key elements in one illustration. It shows a simple current pulse propagating along the transmission line to the point where it has been decided to model the PD and beyond. It shows the partial discharge event being triggered when the field strength exceeds a predetermined level where we wish this to occur; it shows a sourcegenerated sinusoidal signal, representing a power signal (the ripples along the edge near the time axis); and it shows an injected impulse, at the source (left) end.

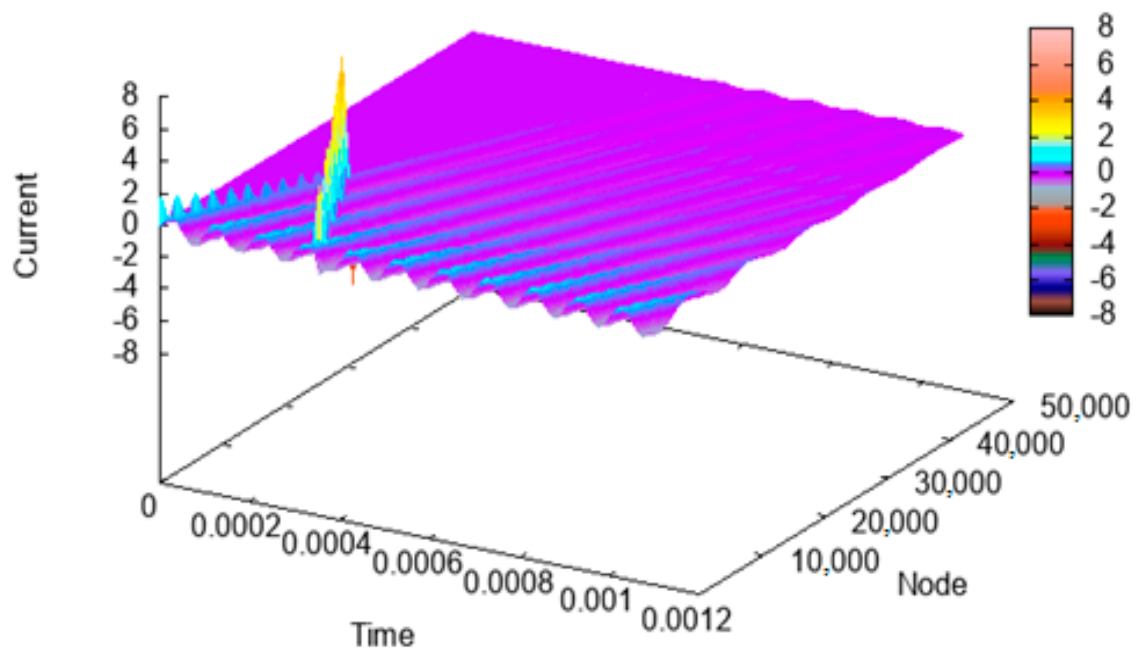

Figure 13. Combining the key elements: a propagating sine wave, an impulse at the source end, and a partial discharge event being triggered within the transmission line. 
The vertical scales are for emphasis, rather than for precision of the physics; they demonstrate that the algorithm works, and make the effects clear, whereas a small partial discharge on a medium voltage (MV) sine wave would be difficult to distinguish in a 3D plot.

\section{Radiation from Partial Discharge Pulses}

Given that the approach appears well behaved for simple propagation studies, a further component to the model is the prediction of radiation. To demonstrate this, a BiotSavart approach was used, which assumed that the current return is sufficiently remote as to not affect the simulation of emissions.

Figure 14 shows the basic configuration, with the transmission line comprising each individual segment, and the current in the segment contributing a small element of magnetic field strength at the point of measurement $P$; the overall contribution is obtained by summing the individual components from each segment at each time step. The time domain magnetic field, $\mathbf{H}$, can then be Fourier-transformed to obtain the frequency domain behavior.

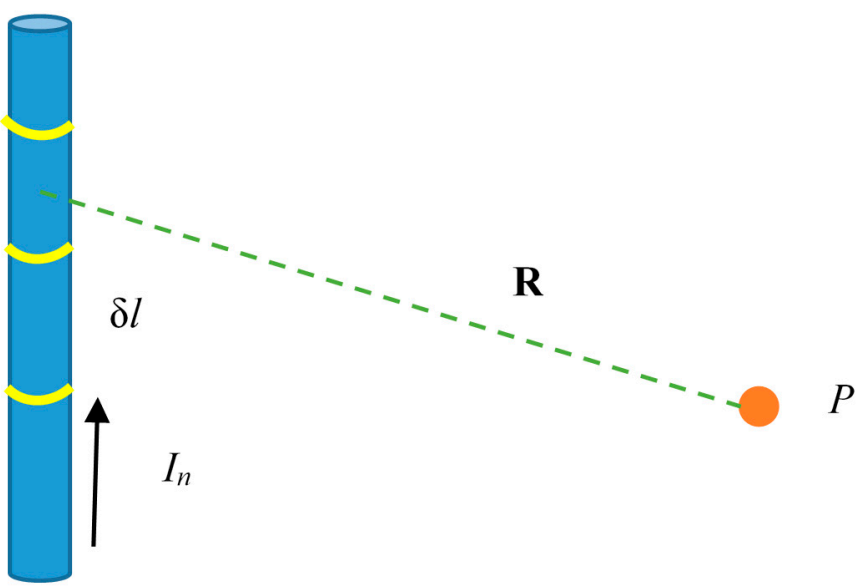

Figure 14. Element of the conductor showing the basic configuration used for analysis.

The nth segment will carry current $I_{n}$, and if the segment length is $\delta 1$ and it is located at the vector $\mathbf{R}$ from the measurement point, the field from each segment can be found from Equation (12) assuming a simple Biot-Savart approach:

$$
\delta \mathbf{H}_{\mathbf{n}}=\frac{\mathrm{I}_{\mathrm{n}} \delta \mathbf{I}_{\mathrm{n}} \times \mathbf{R}_{\mathbf{n}}}{4 \pi\left|\mathbf{R}_{\mathbf{n}}{ }^{3}\right|}
$$

where $\delta \mathbf{H}_{\mathbf{n}}$ is the magnetic field generated by the current in a segment $\delta 1$ of the line and $\delta \mathbf{l}_{\mathrm{n}}$ is a vector with a length $\delta 1$ and direction defined by the current. This can be obtained from each node after the scatter phase, and then combined at each time step according to Equation (13), where $\mathbf{N}$ is the total number of segments in the transmission line and $\mathbf{H}$ is the total magnetic field.

$$
\mathbf{H}=\sum_{\mathbf{n}=1}^{\mathbf{N}} \delta \mathbf{H}_{\mathrm{n}}
$$

While this can be thought of as a simplistic approach, it does provide an introduction to this aspect of the model.

\section{Conducted and Radiated Emission from a Partial Discharge in a MV Transmission Line}

In this section, an illustration of the use of the proposed workbench is given, considering a PD event occurring in a medium voltage (MV) line and evaluating the propagation of the conducted and radiated emission produced by the PD. When, inside the cable insulator of the line, the field strength exceeds a predetermined level, where we wish PD to occur, high frequency pulses start to propagate along the line towards the cable ends. In the 
following sections, the propagation along the line of the PD-generated voltage and current pulses are described and the magnetic field generated by the current pulse is evaluated.

\subsection{Conducted PD Disturbance Propagation}

The 1D TLM model, described in Section 3, is used to describe the PD pulses propagation on a MV power line. A scheme of the system under analysis is shown in Figure 15.

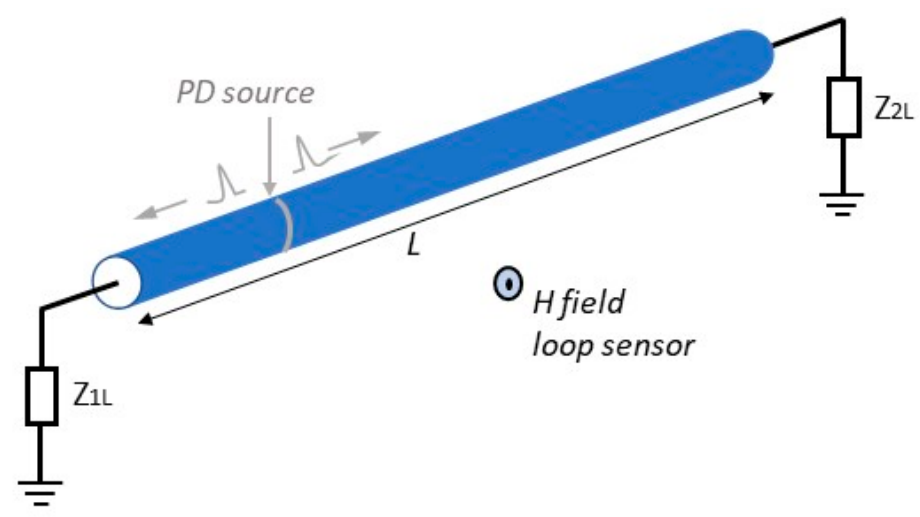

Figure 15. System under analysis.

A line length of $100 \mathrm{~m}$ has been considered, with the PD source located $40 \mathrm{~m}$ from the left end of the cable. The line is formed by a medium voltage coaxial cable with cross-linked polyethylene (XLPE) insulation and a cross-sectional area of $150 \mathrm{~mm}^{2}$. The line is connected, at both ends, to two power transformers, with $Z_{1 L}$ and $Z_{2 L}$ impedance [12].

$\mathrm{PD}$ pulses have high frequency signals with a frequency content up to some $\mathrm{GHz}$; therefore, the TLM model has been developed defining a $\Delta \mathrm{t}=1 / \mathrm{f}_{\max }=1 \cdot 10^{-10} \mathrm{~s}$ to give a maximum frequency of interest, $\mathrm{f}_{\max }=10 \mathrm{GHz}$.

The cable used for the analysis [12] is characterized by the following parameters:

$$
\begin{gathered}
\mathrm{u}=\frac{1}{\sqrt{\mathrm{lc}}}=1.675 \cdot 10^{8} \mathrm{~m} / \mathrm{s} \\
\mathrm{Z}_{0}=\sqrt{\frac{1}{\mathrm{c}}}=15.50 \Omega \\
\mathrm{r}=0.206 \cdot 10^{-3} \Omega / \mathrm{m} \\
\mathrm{g}=1 \cdot 10^{-13} \mathrm{~S} / \mathrm{m}
\end{gathered}
$$

where $\mathrm{u}$ is the propagation speed, $\mathrm{Z}_{0}$ is the characteristic impedance, and $\mathrm{r}, \mathrm{l}, \mathrm{c}$, and $\mathrm{g}$ are the electrical characteristic of the cables per unit length.

The value of $Z_{1 L}$ and $Z_{2 L}$, at the cable ends, is of the order of tens of $k \Omega$, which is the input impedance of power transformers at the high frequency. Therefore, the reflection coefficient of the line given by:

$$
\Gamma=\frac{Z_{\mathrm{L}}-\mathrm{Z}_{0}}{\mathrm{Z}_{\mathrm{L}}+\mathrm{Z}_{0}}
$$

With, $Z_{L}$, the impedance at the cable end is close to 1 , and the PD signals are reflected by the cable end impedances.

Figure 16 shows the PD voltage and current signals at $12 \mathrm{~m}$ from the left end of the cable. The figure shows the PD signal and its reflections from the cable ends and the attenuation of the signals due to the lossy line. The distortion effect, caused by the frequency dependence of the cable parameters, does not appear because it has not been reproduced in this first design of the simulation workbench under development. 

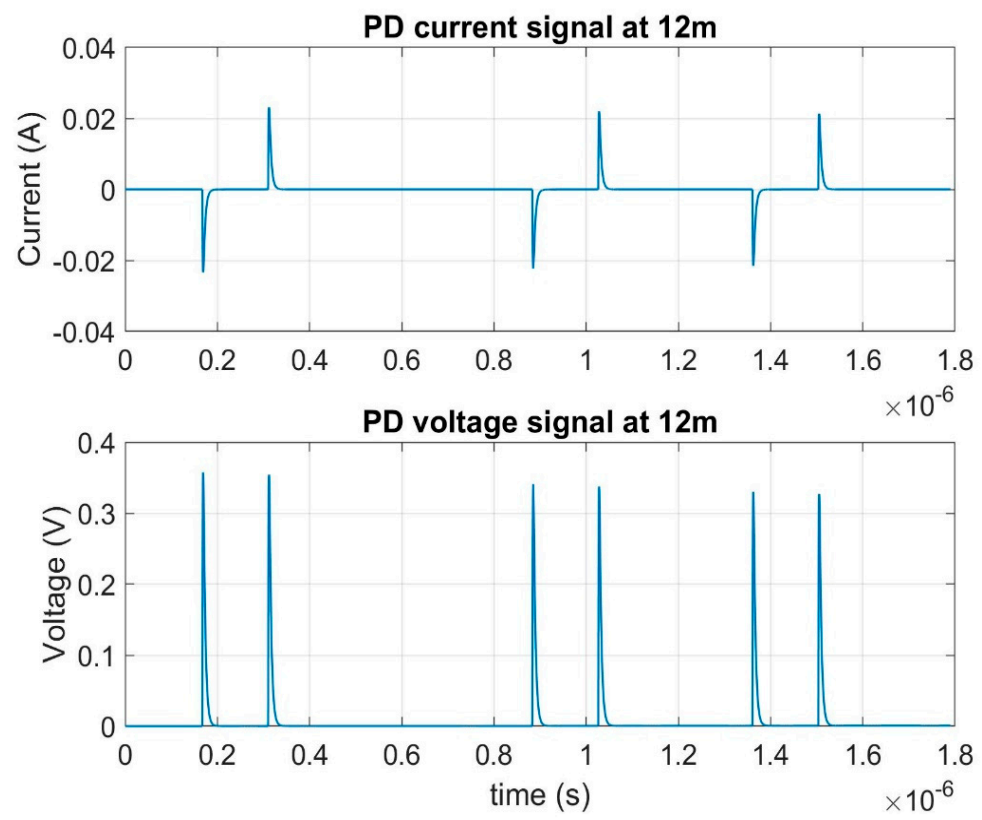

Figure 16. PD voltage and current signals evaluated at $12 \mathrm{~m}$ from the left cable end.

Figure 17 shows the PD voltage and current signals at $40 \mathrm{~m}$ from the left end of the cable, where the PD event occurs. The figure shows the voltage and current signals generated by the PD event and the reflected signals from the right and left end of the cable. At $t=1.2 \mu \mathrm{s}$, the reflected current and voltage signals add up in phase.
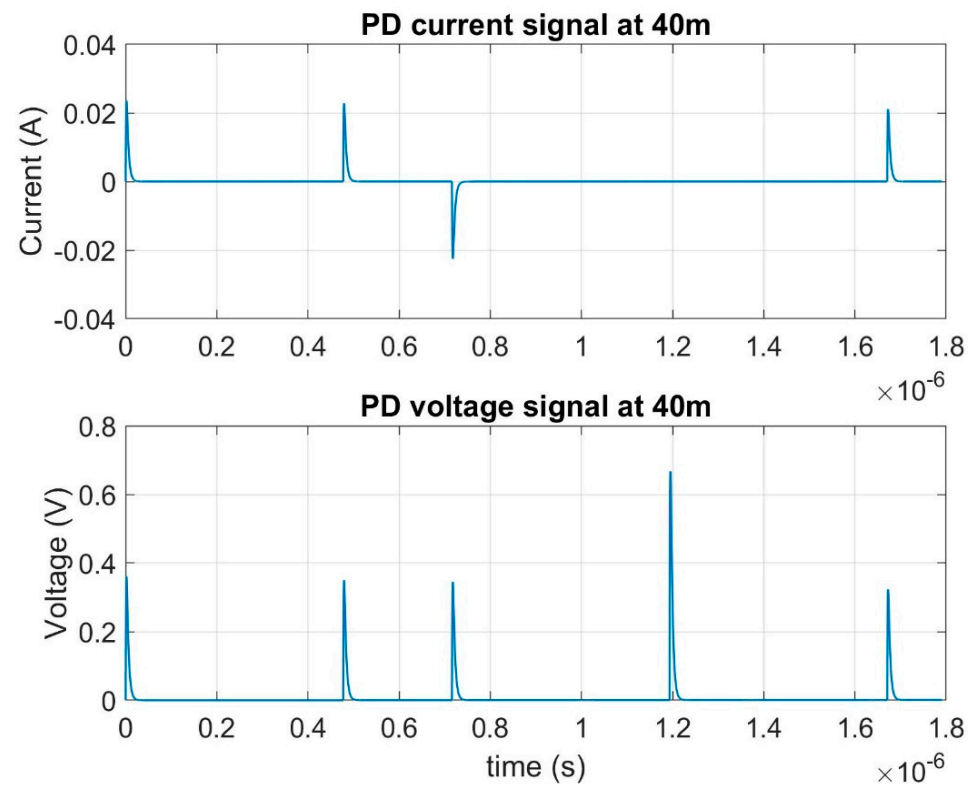

Figure 17. PD voltage and current pulses at the PD location, $40 \mathrm{~m}$ from the left end of the cable.

The measurement of PD signals in multiple observation points of a power network are used in reflectometry or traveling wave-based techniques $[13,14]$ for the on-line location of PD sources, as mentioned in the introduction. The traveling wave-based techniques are based on the principle that PD events produce electromagnetic signals that travel in either direction towards the cable ends, and the measurements of the incident and the reflected signals are used to determine the source location. Then, the proposed TLM workbench, describing both the PD source and the propagation of the generated signals, can be useful to design on-line PD location methods [7].

Finally, Figure 18 shows the Fourier transform of the PD voltage signal at the PD source. 


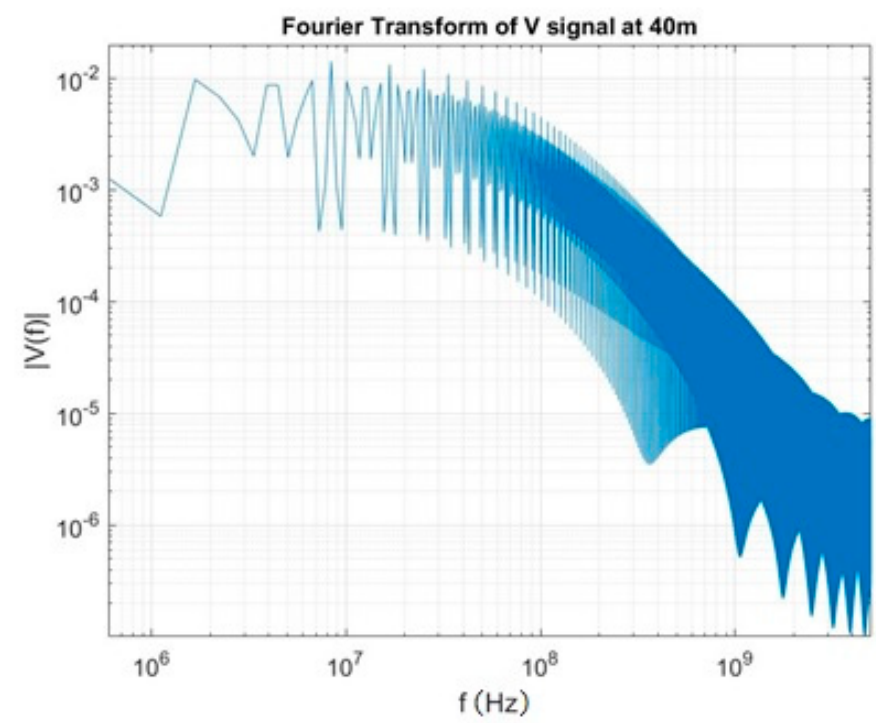

Figure 18. Fourier transform of the PD voltage signal at the PD source.

As the figure shows, the highest frequency content is within hundreds of $\mathrm{MHz}$, and contains frequencies also in the ultra-high frequency (UHF) range, 300-3000 MHz. The ultra-wide band PD diagnosis of power cable accessories [11] is based on the measurement of the UHF components of PD signals that have the advantage of a higher signal-to-noise ratio compared to the IEC 60,270 conventional measurement system [11]. The proposed numerical workbench can help also to design the suitable UHF sensors adopted in the UHF PD diagnosis.

\subsection{Radiated PD Disturbance Propagation}

The $\mathrm{H}$ field produced by the high frequency current signal generated by the PD event is evaluated using the Biot-Savart law, as described in Section 4.

In particular, the circular cylindrical coordinate system is considered as shown in Figure 19.

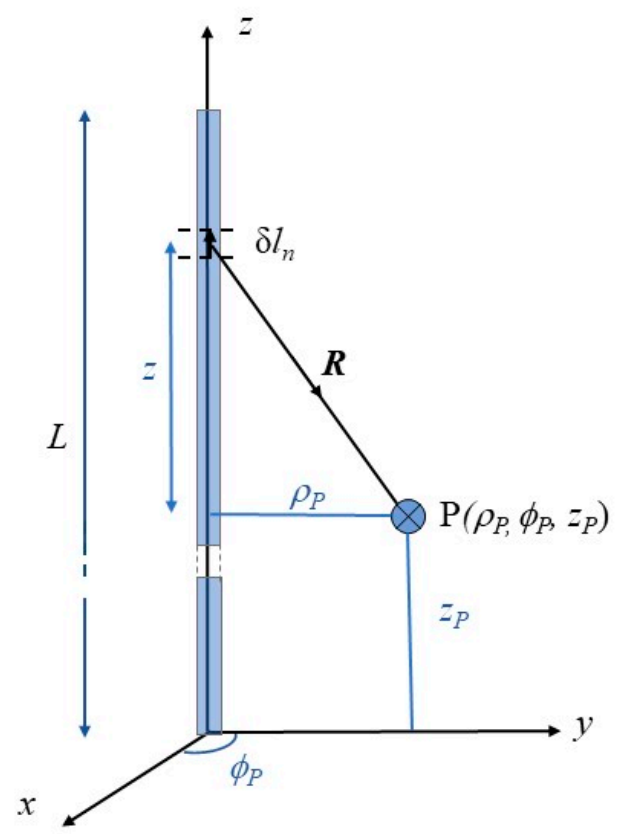

Figure 19. Circular cylindrical coordinate system used in the system under study to evaluate the magnetic field. 
An $\mathrm{H}$-field loop sensor is located in the $z-y$ plane at the point $\mathrm{P}\left(\rho_{\mathrm{P}}, \phi_{\mathrm{P}}, \mathrm{z}_{\mathrm{P}}\right)$, then $\phi_{\mathrm{P}}=\pi / 2$, while the cable is located along the $z$-axis with the left end of the cable at the origin. Considering the positive version of the current signal from the left end of the cable to the right end of the cable, each $\delta l_{n}$ of the $\mathrm{N}$ segments in which the line has been discretized by the TLM method, contributes $\delta \mathbf{H}_{\mathbf{n}}$ to the magnetic field, defined by Equation (12) at each time-step k. From Figure 20, considering that:

$$
\begin{gathered}
\delta 1=\delta l \mathbf{a}_{\mathrm{z}} \\
\mathbf{R}=\rho \mathbf{a}_{\rho}-\mathrm{z} \mathbf{a}_{\mathrm{z}}
\end{gathered}
$$

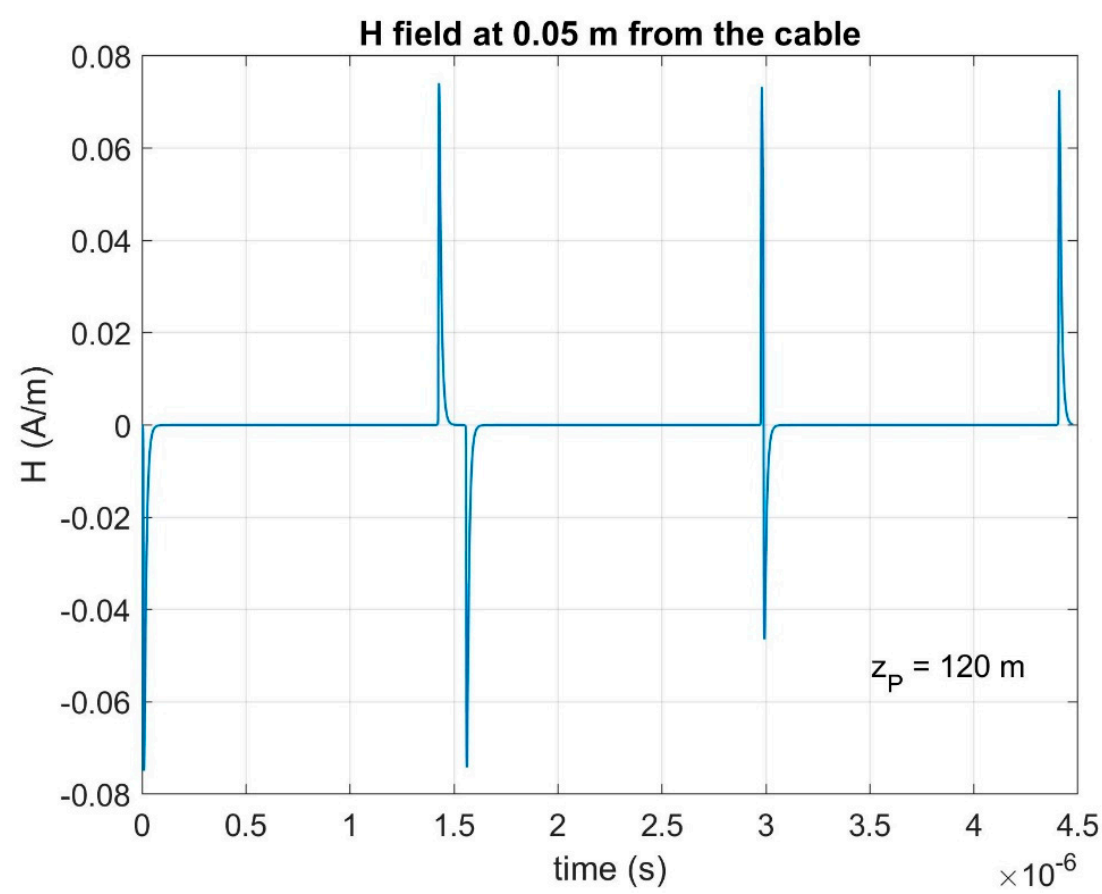

Figure 20. $\mathrm{H}$ field at a distance $0.05 \mathrm{~m}$ from the cable at point $\mathrm{P}$ with $\mathrm{z}_{\mathrm{P}}=38 \mathrm{~m}$, close to the PD source.

Then:

$$
\delta \mathbf{l} \times \mathbf{R}=\rho \delta 1 \mathbf{a} \phi
$$

With:

$$
\begin{gathered}
|\mathbf{R}|=\sqrt{\rho^{2}+\mathrm{z}^{2}} \\
\mathrm{z}=\mathrm{n} \cdot \mathrm{dl}-\mathrm{z}_{\mathrm{p}}
\end{gathered}
$$

where $\mathrm{n}$ is the $\mathrm{n}_{\text {th }}$ segment of the line and $\mathbf{a}$ is the unit vector.

Therefore, for each $\delta l_{n}$ segment of the line:

$$
\delta \mathbf{H}_{\mathbf{n}}=\frac{\mathrm{I}_{\mathrm{n}}(\mathrm{k}) \rho \delta 1}{\left.4 \pi[] \rho^{2}+\mathrm{z}^{2}\right]^{3 / 2}} \mathbf{a} \phi
$$

The value of $\delta 1$ is defined within the discretization procedure of the TLM method and it is given by:

$$
\delta 1=\mathrm{u} \delta \mathrm{t}
$$

where $\mathrm{u}$ is the propagation speed of the signal in the cable under study, defined by Equation (14), and $\delta \mathrm{t}$ is the discretization time defined for the TLM $\left(10^{-10} \mathrm{~s}\right.$ in our case). Finally, at each step-time, $\mathrm{k}$, the total magnetic field at the point $\mathrm{P}$ is given by Equation (13). 
The magnetic field is measured at a point $\mathrm{P}$ at a distance $\mathrm{z}_{\mathrm{P}}=38$ from the right end of the cable, close to the PD source located at $40 \mathrm{~m}$, and at a distance from the cable $\rho_{\mathrm{P}}=0.05 \mathrm{~m}$. The measured magnetic field is shown in Figure 20.

Figure 21 shows the Fourier transformation of the $\mathrm{H}$ field that describes its behavior in the frequency domain. As expected, Figure 21 shows that the PD radiated emission covers the UHF range. UHF sensors are, in fact, used to detect PD radiated emissions in substations [6], then the ToA, time of arrival, method is used to locate PD sources.

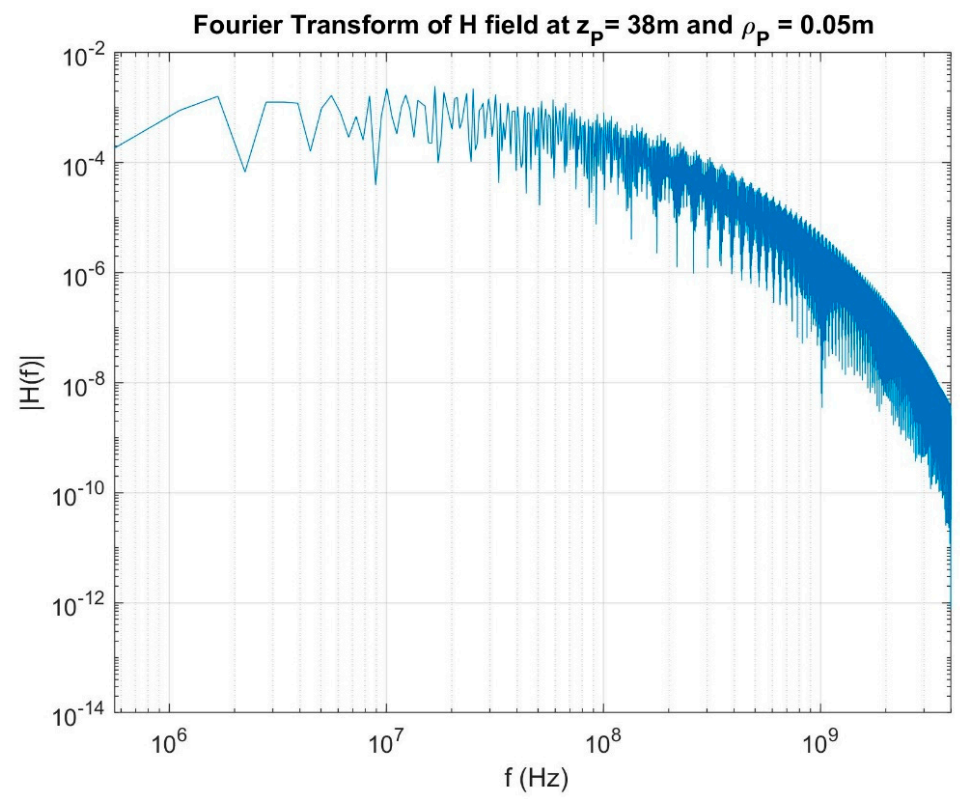

Figure 21. Fourier-transform of the $\mathrm{H}$ field at a distance $0.05 \mathrm{~m}$ from the cable in a point $\mathrm{P}$ with $\mathrm{z}_{\mathrm{P}}=38 \mathrm{~m}$, close to the PD source.

Finally, Figure 22 shows the magnetic field ( $\mathrm{H}$ field) in at the point $\mathrm{P}$ at a distance $\mathrm{z}_{\mathrm{P}}=38 \mathrm{~m}$ from the left end to the cable, when the distance from the cable, $\rho_{\mathrm{P}}$, changes from $0.05 \mathrm{~m}$ to $0.5 \mathrm{~m}$.

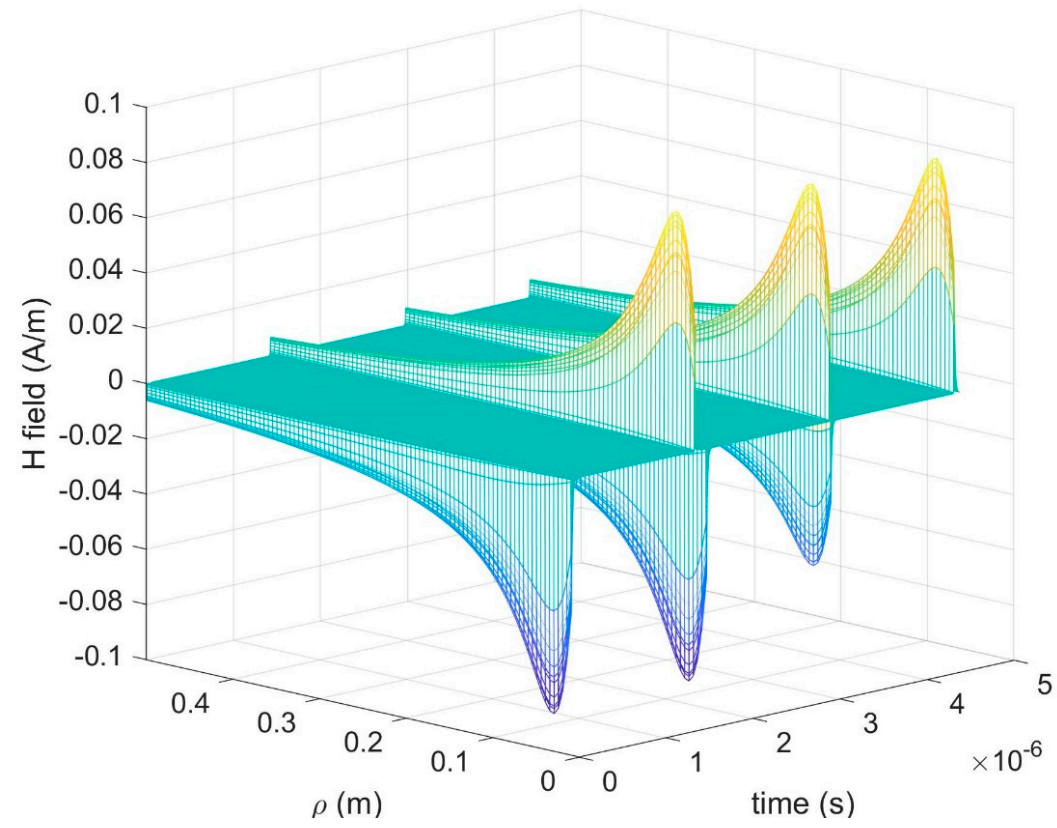

Figure 22. $\mathrm{H}$ field in the point $\mathrm{P}$ at $\mathrm{z}_{\mathrm{P}}=38 \mathrm{~m}$, close to the PD source, and at different distance, $\rho$, from the cable. 


\section{Conclusions and Future Works}

The paper presents the first results of the design of a numerical "workbench", useful to describe partial discharge (PD) events on power networks and to analyze, at the same time, both the conducted and radiated electromagnetic disturbances generated by PDs. An analysis has been developed in order to define the most suitable modeling method to describe PD events in a transmission line context. As a result, the TLM method has been identified as a good and suitable modeling approach to this aim. Hence, a TLM model, describing a PD event in a transmission line context and the propagation of the PD current and voltage signals along the cables, has been developed. The Biot-Savart law is proposed and used to evaluate the magnetic field generated by the high frequency current signals produced by PDs. Finally, an illustration of the use of the proposed workbench to analyze the electromagnetic disturbances generated by a PD event has been given, considering a PD event occurring in a medium voltage (MV) line and evaluating the propagation of the conducted and radiated emission produced by the PD. The numerical workbench can be useful to design methods to locate PD sources on power networks and sensors for the PD detection, key topics to enhance the reliability and resilience of modern power grids. The design is at an early stage and improvements are needed, particularly in modeling the propagation of the high frequency signals generated by the PD source. PD signals are, in fact, subject to distortion due to the skin effect in power cables, and the signal distortion affects the accuracy of PD location methods. An improvement of the model of PD signal propagation on power networks is under development, using the TLM method, to describe both the PD signal distortion and complex power network topologies (inhomogeneous cables [25] and branched lines). The TLM workbench, at the stage presented in this paper, has been already useful in the design of a new PD location method in distribution and transmission networks based on electromagnetic time reversal [7] theory, which is under experimental validation in a real MV network with satisfactory results.

Author Contributions: Conceptualization, A.D.; methodology; A.R., A.D., and H.S.; software, A.R. and H.S.; validation, A.R. and H.S.; formal analysis, A.R. and H.S.; investigation, A.R., A.D., and H.S.; resources, A.D. and A.R.; data curation, A.R.; writing—original draft preparation, A.R., and A.D.; writing - review and editing, A.D. and H.S.; visualization, A.R., A.D. and H.S.; supervision, A.D.; funding acquisition, A.D. and A.R. All authors have read and agreed to the published version of the manuscript.

Funding: This project has received funding from the European Union's Horizon 2020 Research and Innovation Programme under the Marie Skłodowska-Curie grant agreement No. 838681.

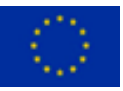

Institutional Review Board Statement: Not applicable.

Informed Consent Statement: Not applicable.

Data Availability Statement: The data presented in this study are openly available in https:// figshare.dmu.ac.uk/ at 10.21253/DMU.13658696.

Acknowledgments: This work was supported by the State Key Laboratory of Electrical Insulation and Power Equipment, Xi'an Jiaotong University, China (EIPE14201).

Conflicts of Interest: The authors declare no conflict of interest. 


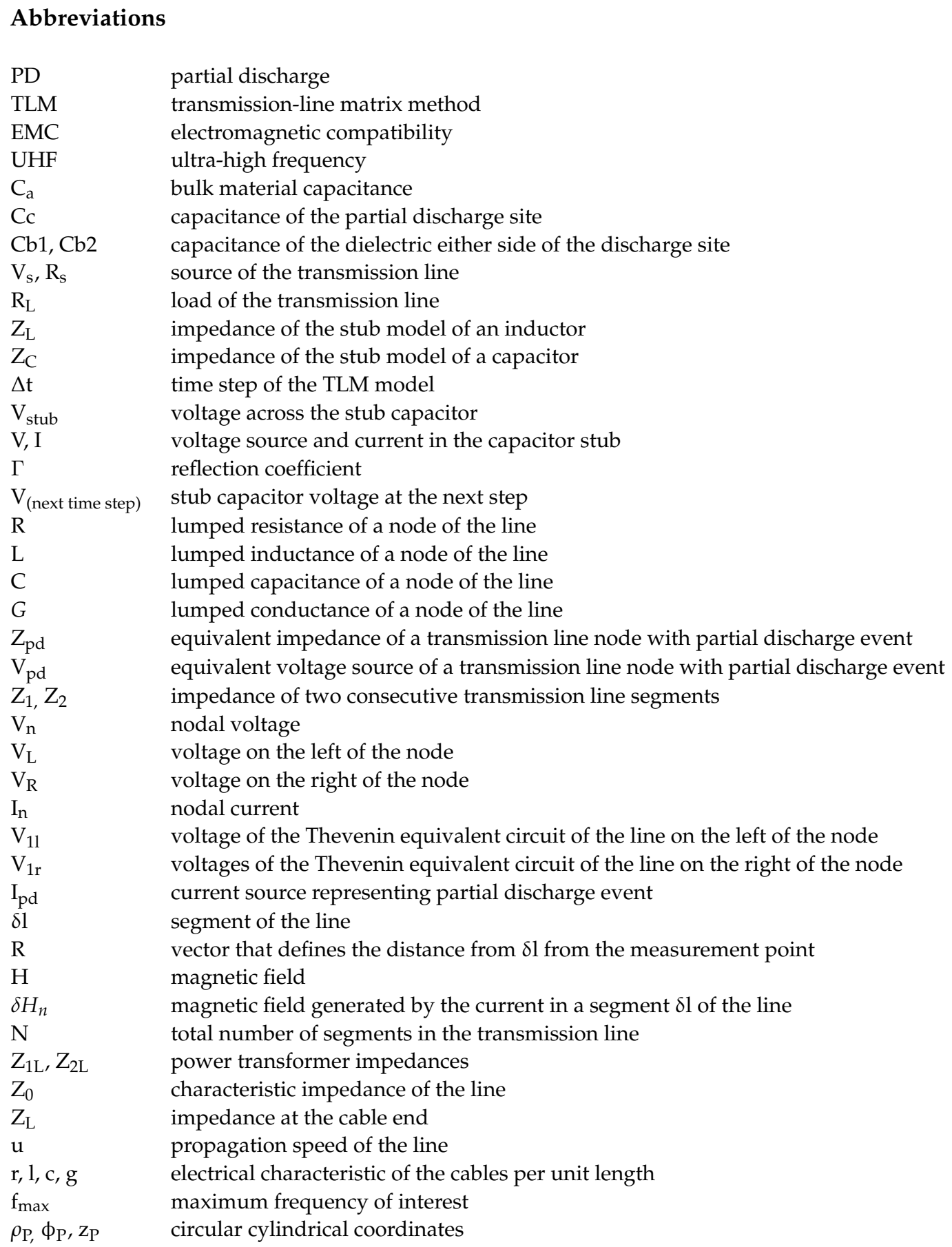

\section{References}

1. Lei, Z.; Song, J.; Tian, M.; Cui, X.; Li, C.; Wen, M. Partial discharge of cavities in ethylene propylene rubber insulations. IEEE Trans. Dielectr. Electr. Insul. 2014, 21, 1647-1659. [CrossRef]

2. Ambikairajah, R.; Phung, B.T.; Ravishankar, J. The modeling of partial discharge waveforms in power systems equipment. In Proceedings of the 2012 Conference on Power and Energy Conference, Ho Chi Min City, Vietnam, 12-14 December 2012.

3. Refaat, S.; Sham, M. A Review of Partial Discharge Detection, Diagnosis Techniques in High Voltage Power Cables. In Proceedings of the 2018 IEEE 12th International Conference on Compatibility, Power Electronics and Power Engineering, Doha, Qatar, 10-12 April 2018.

4. Mohamed, F.; Siew, W.; Liu, K. A Novel Approach in the Measurement of High Frequency Disturbances from Very Large Electrical Systems. IEEE EMC Mag. 2014, 3, 46-50. [CrossRef]

5. Foxall, M.; Duffy, A.; Gow, J.; Seltzer-Grant, M.; Renforth, L. Development of a New High Current Hybrid "Ferrite-Rogowski" Frequency Current Transformer for Partial Discharge Sensing in Medium and High Voltage Cabling. In Proceedings of the International Wire and Cable Symposium, Charlotte, NC, USA, 7-10 November 2010. 
6. Cheng, J.; Xu, Y.; Ding, D.; Liu, W. Investigation of the UHF partial discharge detection characteristics of a novel bushing tap sensor for transformers. IEEE Trans. Power Deliv. 2020, 1-9. [CrossRef]

7. Ragusa, A.; Sasse, H.; Duffy, A.; Rachidi, F.; Rubinstein, M. Electromagnetic Time Reversal Method to Locate Partial Discharges in Power Networks using 1D TLM modelling. IEEE Lett. Electromagn. Compat. Pract. Appl. 2020, 1-5. [CrossRef]

8. Yii, C.C.; Rohani, M.N.K.H.; Isa, M.; Hassan, S.I.S. Multi-end PD Location Algorithm using Segmented Correlation and Trimmed Mean Data Filtering Techniques for MV Underground Cable. IEEE Trans. Dielec. Elect. Insul. 2017, 24, 92-98. [CrossRef]

9. Mohamed, F.P.; Siew, W.H.; Soraghan, J.J.; Strachan, S.M.; McWilliam, J. Partial Discharge Location in Power Cables using a Double Ended Method Based on Time Triggering with GPS. IEEE Trans. Dielec. Elect. Insul. 2013, 20, $2212-2221$.

10. Dukanac, D. Application of UHF method for partial discharge source location in power transformers. IEEE Trans. Dielectr. Electr. Insul. 2018, 25, 2266-2278. [CrossRef]

11. Klein, T.; Denissov, D.; Köhler, W.; Tenbohlen, S. Application of UHF method for on-line PD diagnostics of cable terminations. CIGRE 2008, 2008, 1-8.

12. Zhang, W.; An, B.; Cui, X.; Li, J.; Shi, Y. A Study on Electromagnetic Disturbance in Substation to Wireless Sensor Unit; EMC Europe: Rome, Italy, 2012.

13. Shan, Q.; Glover, I.A.; Atkinson, R.C.; Bhatti, S.A.; Portugues, I.E.; Moore, P.J.; Rutherford, R.; Vieira, M.D.F.Q.; Lima, A.M.N.; De Souza, B.A. Estimation of Impulsive Noise in an Electricity Substations. IEEE Trans. Electr. Compat. 2011, 53, 653-663. [CrossRef]

14. Taccini, A.; Grossi, D.; Vincetti, L.; Maini, M.; Serra, S.; Fattori, M.; Sandrolini, L. Electromagnetic Analysis of PD Detection in GIS Systems; Asia Pacific Symposium on EMC: Singapore, 2012.

15. Illias, G.H.A.; Chen, G.; Lewin, P.L. Comparison between Three-Capacitance, Analytical-based and Finite Element Analysis Partial Discharge Models in Condition Monitoring. IEEE Trans. Diel. Electr. Insul. 2017, 24, 99-109. [CrossRef]

16. Arief, Y.Z.; Izzati, W.A.; Adzis, Z. Modeling of Partial Discharge Mechanisms in Solid Dielectric Material. Inter. J. Eng. Innov. Tech. 2012, 1, 315-320.

17. Niemeyer, L. A generalized Approach to Partial Discharge Modeling. IEEE Trans. Dielec. Elec. Insul. 1995, 2, 510-528. [CrossRef]

18. Ragusa, A.; Sasse, A.H. Duffy—3D Model of Partial Discharge in Defects with Different Sizes and Positions inside Cables for Power Distribution and Transmission Networks. IET Sci. Measurement Technol. 2020, 14, 776-783. [CrossRef]

19. Li, Y.; Wu, L.; Wouters, P.A.; Wagenaars, P.; van der Wielen, P.C.; Steennis, E.F. Effect of ground return path on partial discharge signal propagation along single-core and three-core power cables. Int. Trans. Electr. Energ. Syst. 2016, 26, 1783-1798. [CrossRef]

20. Shafiq, M.; Kauhaniemi, K.; Robles, G.; Hussain, G.A.; Kumpulainen, L. Partial Discharge Signal Propagation in Medium Voltage Branched Cable Feeder. IEEE Electr. Insul. Mag. 2018, 34, 10-29. [CrossRef]

21. Sheng, B.; Zhou, C.; Hepburn, D.M.; Dong, X.; Peers, G.; Zhou, W.; Tang, Z. Partial Discharge Pulse Propagation in Power Cable and Partial Discharge Monitoring System. IEEE Trans. Dielectr. Electr. Insul. 2014, 21, 948-956. [CrossRef]

22. Christopoulos, C. The Transmission Line Modeling Method-TLM, IEEE/OUP Series on Electromagnetic Wave Theory; IEEE Press: Piscataway, NJ, USA, 1995.

23. HA, I. Measurement and Simulation of Partial Discharges within a Spherical Cavity in a Solid Dielectric Material. Ph.D Thesis, University of Southampton, Southampton, UK, 2011.

24. Fung, K.; Hui, S. Fast Simulation of Multistage Power Electronic Systems with Widely Separated Operating Frequencies. IEEE Trans. Power Electr. 1996, 11, 405-412. [CrossRef]

25. Ragusa, A.; Sasse, H.; Duffy, A. Electromagnetic Time Reversal to Locate Partial Discharges in Power Networks with Inhomogeneous cables using the Transmission Line Matrix Method. CIGRE Sci. Eng. J. 2021. Submitted. 